University of Windsor

Scholarship at UWindsor

2010

\title{
The Relationship Among Athlete Leadership Behaviors and Cohesion in Team Sports
}

Diana Vincer

Todd M. Loughead

University of Windsor

Follow this and additional works at: https://scholar.uwindsor.ca/humankineticspub

Part of the Kinesiology Commons

\section{Recommended Citation}

Vincer, Diana and Loughead, Todd M.. (2010). The Relationship Among Athlete Leadership Behaviors and Cohesion in Team Sports. Sport Psychologist, 24 (4), 448-467.

https://scholar.uwindsor.ca/humankineticspub/21

This Article is brought to you for free and open access by the Faculty of Human Kinetics at Scholarship at UWindsor. It has been accepted for inclusion in Human Kinetics Publications by an authorized administrator of Scholarship at UWindsor. For more information, please contact scholarship@uwindsor.ca. 


\title{
The Relationship Among Athlete Leadership Behaviors and Cohesion in Team Sports
}

\author{
Diana J.E. Vincer and Todd M. Loughead \\ University of Windsor
}

\begin{abstract}
This study examined the influence of athlete leadership behaviors on perceptions of team cohesion. The participants were 312 athletes from 25 varsity and club level teams. Each participant completed the Group Environment Questionnaire (Carron, Widmeyer, \& Brawley, 1985) that assessed cohesion and the Leadership Scale for Sports (Chelladurai \& Saleh, 1980) that assessed athlete leadership behaviors. Overall, it was found that individual perceptions of Training and Instruction, and Social Support positively influenced all four dimensions of cohesion (ATG-T, ATG-S, GI-T, GI-S). Furthermore, Autocratic Behavior was negatively associated with the four dimensions of cohesion. Finally, Democratic Behavior was positively related to ATG-T. These findings provide researchers, sport psychology consultants, athletes, and coaches with some initial evidence that it is important to foster the development of athlete leader behaviors to influence the team environment.
\end{abstract}

The construct of cohesion has historically been viewed by some researchers as one of the most important small group variables (e.g., Lott \& Lott, 1965) and is defined as "a dynamic process that is reflected in the tendency for a group to stick together and remain united in the pursuit of its instrumental objectives and/ or for the satisfaction of member affective needs" (Carron, Brawley, \& Widmeyer, 1998, p. 213). As Carron, Bray, and Eys (2002) noted, the definition of cohesion implicitly suggests that higher levels of cohesion are related to greater team performance. In fact, a meta-analysis examining the strength of the cohesion-performance relationship found a moderate to large effect size between these two constructs in sport (Carron, Colman, Wheeler, \& Stevens, 2002). Given the significance of the cohesion-performance relationship, it is not surprising that Westre and Weiss (1991) emphasized the importance of identifying factors that influence the development of cohesion.

To guide research, Carron (1982) advanced a conceptual model of the factors or antecedents that were hypothesized to influence perceptions of cohesion. The antecedents of the model were classified as environmental, personal, team,

Vincer and Loughead are with the Dept. of Kinesiology, University of Windsor, Windsor, Ontario, Canada. 
and leadership factors. Environmental factors referred to the social and physical characteristics of the team's environment and included aspects such as the nature of the task. Next, personal factors referred to individual factors such as individual ability, personality, motivation, and interpersonal compatibility. Team factors referred to group factors such as team norms, team stability, collective efficacy, and group interactions. The final antecedent was leadership factors and was comprised of leadership behaviors, leadership styles, coach-athlete relationships, and coach-team relationships.

Although a strong argument could be made that each of the four antecedents contained in Carron's (1982) conceptual model are important for the development of cohesion, the current study focused on the antecedent of leadership because it may be one of the most important as it is closely related to group effectiveness (Carron, Hausenblas, \& Eys, 2005). To date, the majority of research examining cohesion and leadership has focused on the leadership behaviors of coaches (e.g., Gardner, Shields, Bredemeier, \& Bostrom, 1996; Jowett \& Chaundy, 2004). It should be noted that this body of research has operationalized leader behaviors using the Leadership Scale for Sports (LSS; Chelladurai \& Saleh, 1980), which assesses five dimensions of leadership behaviors. These are Social Support (i.e., satisfying interpersonal needs of group members), Training and Instruction (i.e., improving the athlete's performance), Positive Feedback (i.e., rewarding good performance), Democratic Behavior (i.e., including group members in the decision process), and Autocratic Behavior (i.e., acting independently in decision making). Taken together, research has found the coaching behaviors of Social Support, Training and Instruction, Positive Feedback, and Democratic Behavior were positively related to both task and social cohesion.

It is not surprising that the majority of research has examined the behaviors of the coach because this individual is responsible for making decisions with respect to several team matters, such as strategy, tactics, and team personnel (Loughead, Hardy, \& Eys, 2006). However, recently, research has highlighted another source of leadership on sport teams, namely the athletes (Glenn \& Horn, 1993; Loughead \& Hardy, 2005; Yukelson, 1997). This concept has been labeled athlete leadership and has been defined as "an athlete occupying a formal or informal role within a team, who influences team members to achieve a common goal" (Loughead et al., 2006, p. 144).

To date, research on athlete leadership has compared the leadership behaviors of coach and athlete leaders, the number of athlete leaders on a team, and the functions and characteristics of athlete leaders. Loughead and Hardy (2005) compared the leader behaviors exhibited by coaches and athlete leaders as perceived by athletes from a variety of interdependent team sports (e.g., ice hockey, soccer, and basketball). The participants evaluated the leadership behaviors of their coaches and athlete leaders using the LSS (Chelladurai \& Saleh, 1980). In general, the results indicated that athletes perceived coaches to demonstrate different leadership behaviors than the athlete leaders. Specifically, athletes perceived that coaches exhibited more Training and Instruction, and Autocratic Behavior than athlete leaders. Conversely, athletes perceived that athlete leaders exhibited greater amounts of Social Support, Positive Feedback, and Democratic Behaviors. These results were important because they provided initial empirical evidence that coaches and athletes fulfilled different leadership roles for their teams. 
In addition to comparing coach and athlete leader behaviors, Loughead and Hardy (2005) also investigated the number of athlete leaders present on sport teams. Glenn and Horn (1993) suggested that teams needed one or two athletes on their team to motivate and direct their teammates. However, using a sample of 238 athletes from 15 teams, Loughead and Hardy (2005) found approximately $27 \%$ of athletes from a team's roster were viewed as providing leadership. This result provided some evidence that athlete leadership was more widespread than initially thought, suggesting that leadership within a team is more than a few athletes assuming a leadership role.

With respect to the functions and characteristics of athlete leaders, Loughead et al. (2006) found that they typically (a) occupied either a formal (i.e., captain or assistant captain) or informal leadership (i.e., athletes other than team captains who become leaders based on their interactions with other team members) role on their team, (b) were veteran members of their respective teams, and (c) had higher athletic ability than most team members. Furthermore, they also found that athlete leaders were involved in (a) task-related functions that assisted the team in achieving their goals and objectives, (b) social-related functions that helped satisfy individual member psycho-social needs, and (c) external-related functions that involved representing the team at meetings and media gatherings. Building on these results, Eys, Loughead, and Hardy (2007) examined the relationship between the number of athlete leaders, calculated by dividing the number of athlete leaders by the total number of athletes on their respective teams, over three leadership functions (task, social, and external) and athlete satisfaction. The results indicated that athletes who perceived an equal amount of leaders across the three leadership functions had a higher level of satisfaction than those who perceived an unequal number of athlete leaders. These findings would tend to indicate that it is important to have an equal number of athlete leaders fulfilling each of the three leadership functions.

Although previous research has examined the functions and characteristics of athlete leaders, the number of athlete leaders on a team, compared coach and athlete leader behaviors, and the relationship between athlete leadership and satisfaction, this body of literature does have its shortcomings. First, the majority of the athlete leadership research has focused on the characteristics and the number of athlete leaders (e.g., Eys et al., 2007; Loughead et al., 2006). However, it is equally important to gain a better understanding of the leadership behaviors of these athletes to determine which leadership behaviors are associated with other variables, such as cohesion (Dupuis, Bloom, \& Loughead, 2006). Using semistructured interviews with team captains, Dupuis et al. found that these athlete leaders attempted to positively influence their team's cohesiveness. Second, while leader behaviors is an antecedent in Carron's (1982) conceptual model, the influence of athlete leader behaviors on cohesion have not been studied concurrently. To date, only coach leader behaviors have been examined in relation to cohesion (e.g., Gardner et al., 1996; Jowett \& Chaundy, 2004; Westre \& Weiss, 1991). While this research has highlighted which dimensions of coaches' leadership behaviors are related to cohesion, one issue to note is that some researchers have collapsed the four dimensions of cohesion into two dimensions (e.g., Gardner et al.; Jowett \& Chaundy). That is, researchers have combined the cohesion dimensions of Individual Attractions to the Group-Task (individual team members' feelings about their personal involvement with the group's task) and Group Integration-Task (individual team 
members' feelings about the similarity, closeness, and bonding on the team around the team's task) into a general task cohesion dimension and have combined the cohesion dimensions of Individual Attractions to the Group-Social (individual team members' feelings about their personal acceptance and social interactions with the team) and Group Integration-Social (individual team members' feelings about the similarity, closeness, and bonding on the team around the group as a social unit) into a general social cohesion dimension. As Carron, Brawley, and Widmeyer (2002) noted, the issue of collapsing the four dimensions of cohesion should be done with caution since the dimensions are conceptually different. Therefore, these authors recommended that researchers avoid collapsing the four dimensions of cohesion to calculate a global or overall score.

Thus, the purpose of the current study was to examine the influence of athlete leadership behaviors on team cohesion. While research has shown that athlete leaders engaged in behaviors designed to positively influence the team's cohesiveness (Dupuis et al., 2006), the findings did not specify which athlete leader behaviors would influence perceptions of team cohesion. Given the lack of research examining athlete leadership behaviors and cohesion, the current study used research that has examined the coach leader behavior-cohesion relationship (i.e., Gardner et al., 1996; Jowett \& Chaundy, 2004; Westre \& Weiss, 1991) to formulate the hypotheses. It was predicted that the leadership behaviors of Training and Instruction, Democratic Behavior, Social Support, and Positive Feedback would be positively related to all four dimensions of cohesion, while the leadership dimension of Autocratic Behavior would be negatively related to cohesion. That is, similar results that have been found in the coaching leadership behavior-cohesion relationship would also be present for athlete leader behaviors because a fundamental objective of leadership is to insure that the demands of the team are satisfied (Carron et al., 2005).

\section{Method}

\section{Participants}

The participants were 312 varsity and club level athletes (130 females and 182 males) from 25 intact sport teams sampled from various communities in the province of Ontario (Canada). The mean age of the participants was 19.21 years $(S D=$ $2.59)$ and had, on average, 2.20 years $(S D=1.65)$ of experience with their current team. The participants had been involved in their current sport for an average of 11.24 years $(S D=4.30)$. Finally, the athletes represented a variety of interdependent team sports that included eight ice hockey teams $(n=133$ athletes $)$, two indoor soccer teams ( $n=23$ athletes), eleven volleyball teams ( $n=115$ athletes), and four basketball teams ( $n=41$ athletes).

\section{Measures}

Cohesion. Cohesion was assessed using the Group Environment Questionnaire (Carron, Widmeyer, \& Brawley, 1985). The Group Environment Questionnaire is the most widely used inventory to assess cohesion in sport. Research using the Group Environment Questionnaire has provided ample evidence that the inventory is internally consistent, and has face, concurrent, predictive, and 
factorial validity (cf. Carron et al., 1998). The Group Environment Questionnaire is an 18-item inventory that measures four dimensions of cohesion. The Individual Attractions to the Group-Task dimension contains four items and examines the individual team member's feelings about his/her personal involvement with the group's task, goals and productivity. An example item is: "I'm happy with how much my team wants to win." The Individual Attractions to the Group-Social dimension consists of five items and assesses an individual's feeling about his/ her acceptance and social interaction with the group. An example item is "Some of my best friends are on this team." The Group Integration-Task dimension is comprised of five items and assesses team member's feelings about the similarity and closeness within the team as a whole around the group's task. An example item is "Our teammates have different goals for how we want the team to play." Finally, the Group Integration-Social dimension consists of four items and examines team member's feelings about the similarity and closeness of the group in regards to their social matters. An example item is "Our team would like to spend time together in the off season." All items are scored on a 9-point Likert scale anchored at 1 (strongly disagree) and 9 (strongly agree). Twelve of the 18 items are negatively worded, and thus were reversed scored before data analysis. The items for each dimension of cohesion are summed and then an average is taken for each dimension. Thus, scores can range from 1 to 9 with higher scores indicating higher perceptions of cohesion. The alpha coefficients for the cohesion dimensions of Group Integration-Task $(\alpha=.71)$ and Group Integration-Social $(\alpha=.72)$ were above the generally accepted criterion level of .70, while Individual Attractions to the Group-Task $(\alpha=.65)$ and Individual Attractions to the Group-Social $(\alpha=.60)$ were slightly below the generally accepted level (Nunnally \& Bernstein, 1994). As noted by Carron, Brawley, et al. (2002) both acceptable and marginally suitable internal consistency values have been reported in previous research for both Individual Attractions to the Group-Task and Individual Attractions to the Group-Social. These authors also pointed out that it is not surprising for a dynamic multidimensional construct such as cohesion to have lower than ideal (i.e., <.70) internal consistency values. Carron, Brawley, et al. suggested that:

Accepting this proposition [cohesion as a multidimensional construct] does not involve accepting the premise that all dimensions are equally present across different groups to the same extent and at the same time in the life of a group. Depending upon when a group is assessed along its continuum of group development, members may or may not have sufficient information to evaluate the cohesiveness represented by a specific scale. (p. 26).

Carron, Brawley, et al. (2002) recommended that researchers can either delete one or two items if it can improve the internal consistency value for the offending cohesion dimension or if it cannot be improved by removing items than researchers can interpret any relationships between the offending cohesion dimension and other variables with caution. In the current study, the removal of items from the Individual Attractions to the Group-Task and Individual Attractions to the Group-Social subscales would not have improved the internal consistency values, therefore, the results involving these two dimensions should be interpreted with some caution. 
Athlete Leader Behaviors. The behaviors of athlete leaders were measured using a modified version of the Leadership Scale for Sports (Chelladurai \& Saleh, 1980). This modified version of the Leadership Scale for Sports assesses the same five dimensions as the original version: Training and Instruction, Positive Feedback, Social Support, Democratic Behavior, and Autocratic Behavior. The modified version has been used in previous athlete leadership research (Loughead $\&$ Hardy, 2005). These authors reported acceptable internal consistency values for the five dimensions and evidence of concurrent validity with varsity and club level athletes. The only modification, as noted by Loughead and Hardy, concerned the stem which preceded the items. In the original version, the stem reads "My coach" whereas in the athlete leader version the stem reads "The athlete leader(s) on my team." The Training and Instruction dimension consists of 13 items and examines the leader's behavior aimed at improving the athlete's performance by facilitating strenuous training. An example item is: "Sees to it that every team member is working to his/her capacity." The Positive Feedback dimension consists of five items and assesses the leader's tendency to reinforce a team member's behavior. An example is: "Compliments a team member for his/her performance in front of others." Next, the Social Support dimension is comprised of eight items and it examines the leader's concern for his/her teammates' welfare. An example item is: "Helps team members with their personal problems." The Democratic Behavior dimension consists of nine items and assesses the extent to which the leader involves their teammates in the decision making. An example item is: "Lets team members decide on the plays to be used in a game." Finally, the Autocratic Behavior dimension consists of five items and assesses behavior that involves the athlete leader's independence in decision-making. An example item is: "Refuses to compromise a point." Answers are provided on a five-point Likert scale anchored at 1 (never) to 5 (always). The items for each dimension of athlete leadership behaviors are summed and then an average is taken for each dimension. Thus, scores can range from 1 to 5 with higher scores reflecting stronger perceptions of athlete leader behavior.

A confirmatory factor analysis was conducted using the data of the current study to examine the factorial validity of a five-factor model (i.e., Training and Instruction, Positive Feedback, Social Support, Democratic Behavior, and Autocratic Behavior) with the paths between factors fixed. In particular, a variance-covariance matrix with maximum likelihood of estimation was used through AMOS 17.0 (Arbuckle, 2008). The chi-square test was statistically significant, $\chi 2(730)=1329.85, p=$ .000 . It should be noted that obtaining a significant chi-square is highly likely with large sample sizes. Consequently, additional fit indices were used to evaluate the model fit. In particular, the fit indices used to test the model fit were the Comparative Fit Index (CFI), Tucker-Lewis Index (TLI), and the Root Mean Square Error of Approximation (RMSEA). These fit indices were recommended by several authors because they have different measurement properties (e.g., Hu \& Bentler, 1998; Jackson, Gillaspy, \& Purc-Stephenson, 2009). When values are close to .95 or greater for CFI and TLI, and close to .06 or lower for RMSEA, the model has a reasonably good fit (Hu \& Bentler, 1999). The five-factor model provided a reasonably good fit to the data, $\mathrm{CFI}=.99$, TLI $=.98$, and RMSEA $=.05$. Furthermore, each dimension of the modified version of the Leadership Scale for Sports demonstrated acceptable internal consistencies: Training and Instruction, $\alpha=.88$; 
Positive Feedback, $\alpha=.84$; Social Support, $\alpha=.86$; Democratic Behavior, $\alpha=.79$, and Autocratic Behavior, $\alpha=.74$ (Nunnally \& Bernstein, 1994).

\section{Procedure}

After receiving approval from the university's research ethics board, a convenience sample of 40 coaches were contacted via telephone to outline the study and request permission to administer the questionnaires to the athletes on their teams. The coaches were contacted becausetheir team competed at a high level of sport. Once the approval from the coaches was obtained $(N=25)$, the researchers met with the athletes and they were given a full description of the study. This type of response rate from coaches is similar to what has been reported in previous studies (e.g., Heuzé, Raimbault, \& Fontayne, 2006; Senécal, Loughead, \& Bloom, 2008). The fifteen coaches who refused access to their athletes indicated a lack of time or did not return telephone messages. Using this convenience sample, all athletes received a letter of information for their records and informed consent was implied by the completion and return of the questionnaires to the researchers. Following this briefing, the athletes completed the Group Environment Questionnaire (Carron et al., 1985) and the modified version of the Leadership Scale for Sports (Chelladurai \& Saleh, 1980) in the team's locker or meeting room following a practice session. The athletes completed the questionnaires near the end of the regular season which allowed for the emergence of athlete leaders and perceptions of team cohesion to develop (Loughead \& Carron, 2004). The completion of the questionnaires took approximately $20 \mathrm{~min}$ to complete and the order of the questionnaires were counterbalanced.

\section{Data Analysis}

The unit of analysis question has been an issue for many years in group dynamics in sport research. That is, what would be the appropriate unit of analysis? Carron, Brawley, et al. (2002) noted that three approaches can be taken. First, the individual team member can be used as the unit of analysis. A second approach is to use the aggregate (i.e., the group mean) as the unit of analysis. The third approach is to use the intact team as the unit of analysis. So the question becomes "Which of these three approaches is best?" Unfortunately, there is no simple answer because this requires both conceptual and statistical consideration. Nonetheless researchers should consider three factors. One is the nature of the research question. Some research questions are best answered with a specific unit of analysis. A second factor to consider concerns the nature of the theory being tested. The third factor is empirical in nature. That is, in some cases, analyses can occur either at the individual or team level, or at both the individual and team levels. In the case of the current study, the nature of the concepts being tested (i.e., athlete leadership and cohesion) and the nature of the research question-is there a relationship between athlete leadership behaviors and cohesion-could be answered at the individual, team, or both the individual and team levels. Given that it was conceptually possible that the individual, team, or both the individual and team levels be examined, a statistical consideration was made to calculate two estimates: intraclass correlation (ICC) and the within group interrater reliability index $\left(r_{\mathrm{wg}(\mathrm{j})}\right)$. 
The ICC estimate corresponds to the amount of variance in individual level responses (i.e., athletes' ratings) that can be explained by team level membership (i.e., what team they are on; Bliese, Halverson, \& Schriesheim, 2002). In addition, Bliese (2000) noted that this estimate is also viewed as a measure of nonindependence (i.e., responses from individuals are dependent upon team membership). ICC is calculated as follows:

$$
\mathrm{ICC}=\left(\mathrm{m}_{\mathrm{sb}}-\mathrm{m}_{\mathrm{sw}}\right) /\left[\mathrm{m}_{\mathrm{sb}}+\left(\left(\mathrm{n}_{\mathrm{g}}-1\right) \mathrm{m}_{\mathrm{sw}}\right)\right]
$$

where $\mathrm{m}_{\mathrm{sb}}$ is the between-group mean square, $\mathrm{m}_{\mathrm{sw}}$ is the within-group mean square, and $\mathrm{n}_{\mathrm{g}}$ is the group size. Values to calculate the ICC were derived from the components of a one-way ANOVA with team membership as the independent variable and the variables of interests (i.e., cohesion and athlete leadership) as the dependent variables (James, 1982). Given that one ICC value is computed for each variable across all teams (in this case, five values were computed for each dimension of athlete leadership behavior and four values for each dimension of cohesion), it should be noted that the average team size value was used seeing as team roster sizes differed. Using the average team size is an acceptable practice for this type of calculation (Bliese, 1998).

It was shown that the independent variable of team membership was a significant predictor of the dependent variable of athlete leadership behaviors and cohesion as indicated by significant $F$ ratios from an ANOVA model (all $p$ values $=.00$ ). The ICC values ranged from .07 to .14 for athlete leadership behaviors. Specifically, the values for each dimension of athlete leadership behavior were .08 for Training and Instruction, .08 for Democratic Behavior, .14 for Autocratic Behavior, .09 for Social Support, and .07 for Positive Feedback. As for cohesion, the ICC values varied between .10 and .35. In particular, the cohesion dimension of Individual Attractions to the Group-Task had a value of .10, .15 for Individual Attractions to the Group-Social, .20 for Group Integration-Task, and .35 for Group IntegrationSocial. A large ICC value (e.g., > .70) indicates a large clustering effect with very little individual variability, whereas a small ICC value (e.g., < .10) indicates a weak clustering effect where there is considerable individual variability within teams (Bliese, 1998). Based on the small ICC values for both the five dimensions of athlete leadership behaviors and the four dimensions of cohesion indicate that little variance can be attributed to differences between teams-suggesting that aggregation of individual scores to the group level may not be appropriate.

The index of agreement $\left(r_{\mathrm{wg}(\mathrm{j})}\right)$ represents the amount of interrater agreement (i.e., similarity in athletes' ratings for each team), and is typically used to determine the appropriateness of aggregating the data to higher levels of analysis (James, Demaree, \& Wolf, 1984). Unlike ICC, the $r_{\mathrm{wg}(\mathrm{j})}$ index is calculated separately for each team. Given that the current study contained 25 teams, 25 separate $r_{\mathrm{wg}(\mathrm{j})}$ values were calculated for each subscale of the Group Environment Questionnaire and the Leadership Scale for Sports and then the average of these values were computed. The $r_{\mathrm{wg}(\mathrm{j})}$ is calculated as follows for each team:

$$
r_{\mathrm{wg}(\mathrm{j})}=\frac{J\left[1-\left(s_{\mathrm{j}}^{2} / \sigma_{\mathrm{E}}^{2}\right] r_{\mathrm{wg}(\mathrm{j})}\right.}{J\left[1-\left(s x_{\mathrm{j}}^{2} / \sigma_{\mathrm{E}}^{2}\right)\right]+\left(s x_{\mathrm{j}}^{2} / \sigma_{\mathrm{E}}^{2}\right)}
$$


where $r_{\mathrm{wg}(\mathrm{j})}$ is the within-group interrater reliability based on $J$ items, $s x^{2}$ is the mean of the observed variances on $J$ items, and $\sigma^{2} \mathrm{E}$ is the expected variances (see James et al. for a detailed description on how to derive the components required to calculate the $r_{\mathrm{wg}(\mathrm{j})}$ index).

The values for the $r_{\mathrm{wg}(\mathrm{j})}$ index ranged from .86 to .96 for athlete leadership behaviors (Training and Instruction, .96; Democratic Behavior, .92; Autocratic Behavior, .86; Social Support, .92; and Positive Feedback, .93), suggesting high agreement and that these teams should have their individual level scores aggregated. While for cohesion, the values ranged from .20 to .70 (Individual Attractions to the Group-Task, .24; Individual Attractions to the Group-Social, .70; Group Integration-Task, .40; and Group Integration-Social, .20), indicating low agreement and these variables should be analyzed at the individual level. Some researchers (e.g., Bliese et al., 2002; George, 1990) have suggested a cut-off value between .60 to .70 when deciding to aggregate the data, noting that this type of criterion level is commonly used for other estimates such as Cronbach's Alpha (Nunnally \& Bernstein, 1994).

Overall, the results demonstrated mixed findings to justify aggregation to the group level. On the one hand, the ICC and $r_{\mathrm{wg}(\mathrm{j})}$ values for cohesion indicated little evidence to justify aggregation and therefore cohesion was modeled at the individual level. On the other hand, there was some support for aggregating athlete leadership behaviors, high $r_{\mathrm{wg}(\mathrm{j})}$ values but low ICC values. Therefore, a decision was made to model athlete leadership behaviors at both the individual and group level by taking the average of all teams.

\section{Results}

\section{Descriptive Statistics}

Overall means and standard deviations were calculated for the four dimensions of cohesion and the five dimensions of athlete leader behaviors across all teams. In terms of cohesion, Individual Attractions to the Group-Social was rated the highest $(M=7.48$ on the 9 -point scale, $S D=1.05)$, followed by Individual Attractions to the Group-Task $(M=7.08, S D=1.44)$, Group Integration-Task $(M=6.78, S D$ $=1.30)$, and Group Integration-Social $(M=6.35, S D=1.66)$. Insofar as athlete leader behaviors are concerned, Positive feedback was rated the highest $(M=4.25$ on the 5-point scale, $S D=.59)$, followed by Social support $(M=3.90, S D=.67)$, Training and instruction $(M=3.62, S D=.56)$, Democratic behavior $(M=3.62, S D$ $=.58)$, and finally, Autocratic behavior $(M=2.50, S D=.74)$.

A summary of the bivariate correlations among the variables can be found in Table 1, which demonstrates that there were significant relationships among all of the variables, except between the athlete leader behavior of Training and Instruction and Autocratic Behavior. In particular, it was shown the cohesion dimensions of Individual Attractions to the Group-Task, Individual Attractions to the GroupSocial, Group Integration-Task and Group Integration-Social were positively associated with the athlete leader behaviors of Training and Instruction, Democratic Behavior, Social Support and Positive Feedback. Furthermore, the four dimensions of cohesion were negatively related to the athlete leader behavior of Autocratic Behavior. 
Table 1 Bivariate Correlations Between Cohesion and Athlete Leader Behaviors

\begin{tabular}{lccccccccc}
\hline & ATG-T & ATG-S & GI-T & GI-S & TI & DB & AB & SS & PF \\
\hline ATG-T & - & $.30^{*}$ & $.57 *$ & $.28^{*}$ & $.32^{*}$ & $.28^{*}$ & $-.13^{*}$ & $.34^{*}$ & $.26^{*}$ \\
ATG-S & & - & $.44^{*}$ & $.52^{*}$ & $.34^{*}$ & $.19^{*}$ & $-.17^{*}$ & $.38^{*}$ & $.24^{*}$ \\
GI-T & & & - & $.57^{*}$ & $.51^{*}$ & $.44^{*}$ & $-.29 *$ & $.52^{*}$ & $.46^{*}$ \\
GI-S & & & & - & $.39 *$ & $.28^{*}$ & $-.29 *$ & $.49^{*}$ & $.35^{*}$ \\
TI & & & & & - & $.53^{*}$ & -.01 & $.53^{*}$ & $.42^{*}$ \\
DB & & & & & & - & $-.19 *$ & $.54^{*}$ & $.50^{*}$ \\
AB & & & & & & & - & $-.26^{*}$ & $-.32^{*}$ \\
SS & & & & & & & & - & $.67 *$ \\
PF & & & & & & & & & - \\
\hline
\end{tabular}

Note. ATG-T = Individual attractions to the group-task, ATG-S = Individual attractions to the groupsocial, GI-T = Group integration-task, GI-S = Group integration-social. TI = Training and instruction, $\mathrm{DB}=$ Democratic behavior, $\mathrm{AB}=$ Autocratic behavior, $\mathrm{SS}=$ Social support, $\mathrm{PF}=$ Positive feedback. $* p<.01$.

\section{Multilevel Modeling}

Multivariate multilevel regression was used to determine if athlete leadership behaviors (modeled at both the individual and group level) influenced individual athletes' perceptions of cohesion using HLM 6 (Raudenbush, Bryk, Cheong, Congdon, \& du Toit, 2004). Given that there were four dimensions of cohesion, a separate model for each dimension was created whereby the level-1 parameters (b coefficients) were able to randomly vary between teams. The individual model was as follows:

Cohesion $=\beta_{0 \mathrm{j}}+\beta_{1 \mathrm{j}}(\text { Training and Instruction })_{\mathrm{ij}}+\beta_{2 \mathrm{j}}(\text { Democratic Behavior })_{\mathrm{ij}}$ $+\beta_{3 \mathrm{j}}(\text { Autocratic Behavior })_{\mathrm{ij}}+\beta_{4 \mathrm{j}}(\text { Social Support })_{\mathrm{ij}}+\beta_{5 \mathrm{j}}($ Positive Feedback $)$ ij $+e_{\text {ij }}$

$b_{0 \mathrm{j}}$ refers to the average cohesion for team $\mathrm{j} ; \beta_{1 \mathrm{j}}$ refers to the relationship between Training and Instruction and perceptions of cohesion; $\beta_{2 j}$ represents the relationship between Democratic Behavior and perceptions of cohesion; $\beta_{3 \mathrm{j}}$ represents the relationship between Autocratic Behavior and perceptions of cohesion; $\beta_{4 j}$ refers to the relationship between Social Support and perceptions of cohesion; $\beta_{5 j}$ represents the relationship between Positive Feedback and perceptions of cohesion; and finally $\mathrm{e}_{\mathrm{ij}}$ represents the residual.

Each regression coefficient at the individual level becomes the dependent variables for the group level model. Therefore, the group level model was as follows:

$\beta_{0 \mathrm{j}}=\gamma_{00}+\gamma_{01}$ (Training and Instruction team $)_{\mathrm{j}}+\gamma_{02}$ (Democratic Behavior team $)_{j}+\gamma_{03}$ (Autocratic Behavior team $)_{j}+\gamma_{04}(\text { Social Support team })_{j}+\gamma_{05}$ $(\text { Positive Feedback team })_{j}+u_{i j}$

$\beta_{0 \mathrm{j}}$ refers to the average perception of cohesion for team $\mathrm{j} ; \gamma_{00}$ refers to the intercept for the group level model; $\gamma_{01}$ represents the relationship between the 
athlete leadership behavior of Training and Instruction and perceptions of cohesion for all teams $\mathrm{j} ; \gamma_{02}$ represents the relationship between the athlete leadership behavior of Democratic Behavior and perceptions of cohesion for teams $j ; \gamma_{03}$ refers to the relationship between the athlete leadership behavior of Autocratic Behavior and the perceptions of cohesion for teams $\mathrm{j} ; \gamma_{04}$ refers to the relationship between the athlete leadership behavior of Social Support and the perceptions of cohesion for teams $\mathrm{j} ; \gamma_{05}$ represents the relationship between the athlete leadership behavior of Positive Feedback and the perceptions of cohesion for teams $j$, and finally $u_{i j}$ is the random effect.

Table 2 shows the results of the multilevel model for the cohesion dimension of Individual Attractions to the Group-Task. It was found that individual perceptions of Training and Instruction, Democratic Behavior, and Social Support positively influenced perceptions of Individual Attractions to the Group-Task $\left(\beta_{1 \mathrm{j}}=.57, p<.01\right.$; $\beta_{2 \mathrm{j}}=.36, p<.05 ; \beta_{4 \mathrm{j}}=.44, p<.01$, respectively). That is, athletes who reported high athlete leadership behaviors perceived high task cohesion. In contrast, the athlete

\section{Table 2 Perceptions of Athlete Leadership Behaviors on Individual Attractions to the Group-Task}

\begin{tabular}{llccccc}
\hline ATG-T & $\begin{array}{l}\text { Fixed } \\
\text { Effect }\end{array}$ & Parameter & Coefficients & SE & T-ratio & \\
\hline Intercept & $\beta_{0 \mathrm{j}}$ & 7.11 & .11 & $67.45^{* * *}$ & \\
TIteam & $\mathrm{g}_{01}$ & -.06 & .49 & -.13 & \\
DBteam & $\mathrm{g}_{02}$ & .48 & .34 & 1.44 & \\
ABteam & $\mathrm{g}_{03}$ & -.48 & .40 & -1.20 & \\
SSteam & $\mathrm{g}_{04}$ & -.18 & .50 & -.36 & \\
PFteam & $\mathrm{g}_{05}$ & -1.30 & .93 & -1.40 & \\
TI & $\beta_{0 \mathrm{j}}$ & .57 & .18 & $3.13^{* *}$ & \\
DB & $\beta_{0 \mathrm{j}}$ & .36 & .15 & $2.46^{*}$ & \\
AB & $\beta_{0 \mathrm{j}}$ & -.29 & .12 & $-2.49 *$ & \\
SS & $\beta_{0 \mathrm{j}}$ & .44 & .14 & $3.10^{* *}$ & \\
PF & $\beta_{0 \mathrm{j}}$ & -.15 & .16 & -.92 & \\
Random & & Variance & & & \\
Effect & Parameter & component & $d f$ & $\chi^{2}$ & Reliability \\
ATG-T & $\sigma^{2}{ }_{\mathrm{u} 0}$ & .25 & 20 & $60.37^{* * *}$ & .65 \\
Residual & $\sigma_{\mathrm{e}}^{2}$ & 1.5 & & & \\
\hline
\end{tabular}

Note. ATG-T = Individual attractions to the group-task, TIteam = team perceptions of Training and instruction, DBteam = team perceptions of Democratic behavior, ABteam $=$ team perceptions of Autocratic behavior, SSteam $=$ team perceptions of Social support, PFteam $=$ team perceptions of Positive feedback. TI $=$ individual perceptions of Training and instruction, DB $=$ individual perceptions of Democratic behavior, $\mathrm{AB}=$ individual perceptions of Autocratic behavior, $\mathrm{SS}$ = individual perceptions of Social support, $\mathrm{PF}=$ individual perceptions of Positive feedback.

$* p<.05, * * p<.01, * * * p<.001$. 
leadership behavior of Autocratic Behavior was shown to negatively influence this dimension of cohesion $\left(\beta_{3 j}=-.29, p<.05\right)$. In other words, athletes perceiving high autocratic behaviors by their athlete leaders perceived lower levels of task cohesion. In contrast, none of the team level athlete leader behaviors were significantly related to the cohesion dimension of Individual Attractions to the Group-Task.

Table 3 highlights the results of the multilevel model for the cohesion dimension of Individual Attractions to the Group-Social. On the one hand, it was found that individual perceptions of Training and Instruction, and Social Support positively influenced perceptions of Individual Attractions to the Group-Social $\left(\beta_{1 \mathrm{j}}=.40\right.$, $p<.01 ; \beta_{4 \mathrm{j}}=.31, p<.05$, respectively). In other words, athletes reporting high athlete leadership behaviors of Training and Instruction, and Social Support also reported high social cohesion. On the other hand, the athlete leader behavior of Autocratic Behavior was negatively associated this dimension of cohesion $\left(\beta_{3 \mathrm{j}}=\right.$ $-.25, p<.001)$. None of the team level athlete leader behaviors were significantly related to Individual Attractions to the Group-Social.

\section{Table 3 Perceptions of Athlete Leadership Behaviors on Individual Attractions to the Group-Social}

\begin{tabular}{llccccc}
\hline \multirow{2}{*}{ ATG-S } & Fixed & & & & & \\
& Effect & Parameter & Coefficients & SE & T-ratio & \\
\hline Intercept & $\beta_{0 \mathrm{j}}$ & 7.41 & .09 & $84.19^{* * *}$ & \\
TIteam & $\mathrm{g}_{01}$ & -.26 & .38 & -.67 & \\
DBteam & $\mathrm{g}_{02}$ & -.22 & .41 & -.53 & \\
ABteam & $\mathrm{g}_{03}$ & -.62 & .34 & -1.79 & \\
SSteam & $\mathrm{g}_{04}$ & .83 & .62 & 1.34 & \\
PFteam & $\mathrm{g}_{05}$ & -1.27 & .80 & -1.60 & \\
TI & $\beta_{0 \mathrm{j}}$ & .40 & .13 & $2.96^{* * *}$ & \\
DB & $\beta_{0 \mathrm{j}}$ & .03 & .13 & .26 & \\
AB & $\beta_{0 \mathrm{j}}$ & -.25 & .06 & $-4.29 * * *$ & \\
SS & $\beta_{0 \mathrm{j}}$ & .31 & .12 & $2.60 *$ & \\
PF & $\beta_{0 \mathrm{j}}$ & -.06 & .12 & -.55 & \\
Random & & Variance & & & Reliability \\
Effect & Parameter & component & $d f$ & $\chi^{2}$ & .73 \\
ATG-S & $\sigma^{2}{ }_{\mathrm{u} 0}$ & .19 & 20 & $77.23 * * *$ & \\
Residual & $\sigma^{2}{ }_{\mathrm{e}}$ & .80 & & & \\
\hline
\end{tabular}

Note. ATG-S = Individual attractions to the group-social, TIteam = team perceptions of Training and instruction, DBteam = team perceptions of Democratic behavior, ABteam = team perceptions of Autocratic behavior, SSteam = team perceptions of Social support, PFteam = team perceptions of Positive feedback. TI = individual perceptions of Training and instruction, DB = individual perceptions of Democratic behavior, $\mathrm{AB}=$ individual perceptions of Autocratic behavior, $\mathrm{SS}$ = individual perceptions of Social support, $\mathrm{PF}=$ individual perceptions of Positive feedback.

$* p<.05, * * p<.01, * * * p<.001$. 
Table 4 shows the results of the multilevel model for the cohesion dimension of Group Integration-Task. It was found that individual perceptions of Training and Instruction, and Social Support $\left(\beta_{1 \mathrm{j}}=.77, p<.001 ; \beta_{4 \mathrm{j}}=.27, p<.05\right.$, respectively) positively influenced Group Integration-Task. While the athlete leadership behavior of Autocratic Behavior $\left(\beta_{3 \mathrm{j}}=-.35, p<.001\right)$ was found to negatively influence Group Integration-Task. Furthermore, none of the team level athlete leader behaviors were significantly related to Group Integration-Task.

Table 5 shows the results of the multilevel model of the cohesion dimension of Group Integration-Social. The results showed that individual perceptions of Training and Instruction, and Social Support $\left(\beta_{1 \mathrm{j}}=.52, p<.01 ; \beta_{4 \mathrm{j}}=\right.$ $.54, p<.01$, respectively) positively influenced this dimension of cohesion. The athlete leader behavior of Autocratic Behavior $\left(\beta_{3 j}=-.42, p<.001\right)$ was found to have a negative impact on Group Integration-Social. In addition, none of the team level athlete leader behaviors were significantly related to Group Integration-Social.

Table 4 Perceptions of Athlete Leadership Behaviors on Group Integration-Task

\begin{tabular}{lccccc}
\hline GI-T & Fixed & & & & \\
Effect & Parameter & Coefficients & SE & T-ratio & \\
\hline Intercept & $\beta_{0 \mathrm{j}}$ & 6.74 & .13 & $53.84 * * *$ & \\
TIteam & $\mathrm{g}_{01}$ & -.48 & .44 & -1.10 & \\
DBteam & $\mathrm{g}_{02}$ & .00 & .47 & .00 & \\
ABteam & $\mathrm{g}_{03}$ & -.57 & .58 & -1.00 & \\
SSteam & $\mathrm{g}_{04}$ & .55 & .58 & .95 & \\
PFteam & $\mathrm{g}_{05}$ & -1.42 & 1.26 & -1.13 & \\
TI & $\beta_{0 \mathrm{j}}$ & .77 & .15 & $5.01^{* * *}$ & \\
DB & $\beta_{0 \mathrm{j}}$ & .22 & .14 & 1.55 & \\
AB & $\beta_{0 \mathrm{j}}$ & -.35 & .10 & $-3.69 * * *$ & \\
SS & $\beta_{0 \mathrm{j}}$ & .27 & .13 & $2.17 *$ & \\
PF & $\beta_{0 \mathrm{j}}$ & .18 & .10 & 1.75 & \\
Random & & Variance & & & \\
Effect & Parameter & component & $D f$ & $\chi^{2}$ & Reliability \\
GI-T & $\sigma^{2}{ }_{\mathrm{u} 0}$ & .44 & 20 & $132.79 * * *$ & .85 \\
Residual & $\sigma_{\mathrm{e}}^{2}$ & .88 & & & \\
\hline
\end{tabular}

Note. GI-T = Group integration-task, TIteam = team perceptions of Training and instruction, DBteam = team perceptions of Democratic behavior, ABteam = team perceptions of Autocratic behavior, SSteam $=$ team perceptions of Social support, $\mathrm{PFteam}=$ team perceptions of Positive feedback. TI $=$ individual perceptions of Training and instruction, $\mathrm{DB}=$ individual perceptions of Democratic behavior, $\mathrm{AB}=$ individual perceptions of Autocratic behavior, $\mathrm{SS}=$ individual perceptions of Social support, $\mathrm{PF}=$ individual perceptions of Positive feedback.

$* p<.05, * * * p<.001$. 


\section{Table 5 Perceptions of Athlete Leadership Behaviors on Group Integration-Social}

\begin{tabular}{|c|c|c|c|c|c|c|}
\hline GI-S & $\begin{array}{l}\text { Fixed } \\
\text { Effect }\end{array}$ & Parameter & Coefficients & SE & T-ratio & \\
\hline & Intercept & $\beta_{0 j}$ & 6.23 & .20 & $31.11 * * *$ & \\
\hline & TIteam & $\mathrm{g}_{01}$ & -.23 & .82 & -.28 & \\
\hline & DBteam & $\mathrm{g}_{02}$ & -.69 & 1.01 & -.68 & \\
\hline & ABteam & $\mathrm{g}_{03}$ & -1.57 & 1.03 & -1.53 & \\
\hline & SSteam & $\mathrm{g}_{04}$ & 1.35 & 1.13 & 1.19 & \\
\hline & PFteam & $\mathrm{g}_{05}$ & -2.17 & 2.20 & -.99 & \\
\hline & TI & $\beta_{0 j}$ & .52 & .19 & $2.67 * *$ & \\
\hline & DB & $\beta_{0 \mathrm{j}}$ & .03 & .14 & .20 & \\
\hline & $\mathrm{AB}$ & $\beta_{0 j}$ & -.42 & .10 & $-4.08 * * *$ & \\
\hline & SS & $\beta_{0 j}$ & .54 & .15 & $3.63 * *$ & \\
\hline & PF & $\beta_{0 j}$ & .07 & .14 & .51 & \\
\hline & $\begin{array}{l}\text { Random } \\
\text { Effect }\end{array}$ & Parameter & $\begin{array}{l}\text { Variance } \\
\text { component }\end{array}$ & $d f$ & $\chi^{2}$ & Reliability \\
\hline & GI-S & $\sigma_{\mathrm{u} 0}^{2}$ & 1.21 & 20 & $203.19 * * *$ & .90 \\
\hline & Residual & $\sigma_{\mathrm{e}}^{2}$ & 1.43 & & & \\
\hline
\end{tabular}

Note. GI-S = Group integration-social. TIteam = team perceptions of Training and instruction, DBteam $=$ team perceptions of Democratic behavior, ABteam $=$ team perceptions of Autocratic behavior, SSteam $=$ team perceptions of Social support, PFteam $=$ team perceptions of Positive feedback. TI $=$ individual perceptions of Training and instruction, $\mathrm{DB}=$ individual perceptions of Democratic behavior, $\mathrm{AB}=$ individual perceptions of Autocratic behavior, $\mathrm{SS}=$ individual perceptions of Social support, $\mathrm{PF}=$ individual perceptions of Positive feedback.

$* * p<.01, * * * p<.001$.

\section{Discussion}

This study examined the influence of athlete leader behaviors on perceptions of cohesion. A series of multivariate multilevel regressions were estimated to test the relationship between athlete leader behaviors and cohesion. On the one hand, it was hypothesized that the athlete leader behaviors of Training and Instruction, Democratic Behavior, Social Support, and Positive Feedback would be positively related to task (Individual Attractions to the Group-Task, Group Integration-Task) and social (Individual Attractions to the Group-Social, Group Integration-Social) dimensions of cohesion. On the other hand, it was predicted that the athlete leader behavior of Autocratic Behavior would be negatively related to both task and social cohesion. The results partially supported these hypotheses that specific behaviors of an athlete leader contribute to specific perceptions of cohesion in sport. Specifically, it was found that all four dimensions of cohesion were positively related to the athlete leader behaviors of Training and Instruction, and Social Support. In addition, all four dimensions of cohesion were negatively related to the athlete 
leader behavior of Autocratic Behavior. Finally, only the cohesion dimension of Individual Attractions to the Group-Task was related to the athlete leader behavior of Democratic Behavior. Beyond these specific findings, a number of aspects associated with the results should be highlighted.

The first aspect pertaining to the results is the positive relationship between athlete leader behaviors and cohesion. Based on the operational definitions of the subscales of cohesion and leadership, the results suggested that athlete leaders who demonstrated leadership behaviors toward improving performance through rigorous training and instruction and showed an increased amount of concern for the team member's welfare had teammates who perceived a higher sense involvement in the productivity of team goals, of personal acceptance and social interactions within their team, of similarity, closeness, and unity within the group around the team's task objectives, and to their team as a social unit (Carron, 1982; Chelladurai \& Saleh, 1980). The results of the present supplement the Dupuis et al. (2006) finding by suggesting that the leadership behaviors of Training and Instruction, and Social Support can positively influence a team's cohesiveness both at a task and social level. Thus, it is important for athlete leaders to use high levels of Training and Instruction, and Social Support behaviors.

A second point pertains to the negative relationship between the athlete leader behavior of Autocratic Behavior and all four dimensions of cohesion. Previous coaching leadership research has shown that this leadership behavior is negatively related to both task and social cohesion (e.g., Gardner et al., 1996). Thus, the athletes' perception of their cohesiveness (i.e., team's productivity toward their goals and their personal acceptance within the team) was lower when they felt their athlete leaders taking a more authoritative role in the decision making process. This negative relationship between Autocratic Behavior and cohesion (task and social) may be viewed from a cohesion perspective as the athletes feeling a decreased sense of closeness, bonding, personal involvement, and personal acceptance with their teammates. Consequently, it would appear that autocratic type of athlete leadership behaviors can detract from a team's cohesiveness.

A surprising finding was that the athlete leader behaviors of Positive Feedback and Democratic Behavior (except for Individual Attractions to the Group-Task) were not related to perceptions of cohesion. Previous coach leadership research has shown that Positive Feedback and Democratic Behavior were related to task and social cohesion (Gardner et al., 1996; Jowett \& Chaundy, 2004; Westre \& Weiss, 1991). In addition, Loughead and Hardy (2005) found that athlete leaders provided more Positive Feedback and Democratic Behavior than coaches. Taken together, it would appear that positive reinforcement originating from the athlete leaders has less of an impact on team members than when coming directly from the coaching staff. It is possible that the team members experience a higher frequency of Positive Feedback from their athlete leaders on a regular basis. Therefore, the importance and significance of the feedback originating from their athlete leaders would have less of an impact than when coaches give them some type of positive encouragement - a possible avenue for future research. As for Democratic Behavior, the results of the current study may be explained by considering the findings from Loughead and Hardy who found that there are multiple athlete leaders providing leadership to team members. It may be plausible that with a large number of athlete leaders, it becomes difficult for the team as a whole to reach a consensus 
on a decision. Consequently, the process of decision making could become disorganized and unproductive with higher levels of democratic behavior. Thus, it may be beneficial for the coaching staff to incorporate democratic behavior within their own leadership roles instead of having the athlete leaders make a decision among themselves. In fact, previous research has suggested that athletes prefer coaches to incorporate democratic behavior when making decisions that have a minor effect on team performance (Chelladurai, 1993).

Team level perceptions of the five athlete leader behaviors were not significantly related to cohesion. The nonsignificant finding could be due to the fact that the individual- and team-level athlete leader behaviors represent two different conceptual constructs. Bliese (2000) noted that this type of relationship is known as the fuzzy composition model. This conceptualization suggests that the aggregate, in this case, team-level athlete leader behaviors, often represent a similar but different construct than the individual-level construct (i.e., individual-level athlete leader behaviors). Thus, in the current study, the aggregate might tap into the athlete leader behaviors of the team as a whole, whereas the individual perceptions may represent perceptions of the behaviors as perceived by the individual team member. Consequently, the absence of a relationship between team-level athlete leader behaviors and cohesion may call into question the validity of the hypothesized team-level construct (Chan, 1998). That is, by using statistics such as the index of agreement to justify aggregation from an individual-level construct to a team-level construct, researchers may not be capturing the conceptually distinct nature of team-level athlete leader behaviors. Instead of aggregating individual team members' perceptions, perhaps the use of a group discussion to capture this teamlevel construct will enable researchers to obtain a more valid estimate of athlete leader behaviors at the team-level. This issue clearly warrants further research and is beyond the scope of the current study.

Another reason why the team level perceptions of athlete leadership were not significantly related to cohesion may be related to the number and types of athlete leaders on a team. As noted in the definition of athlete leadership (Loughead et al., 2006), athlete leaders may occupy a formal or informal leadership role within a team. In addition, research has shown that athlete leaders can also assume a task or social leadership position (Loughead et al.) and that athlete leadership is widespread with $27 \%$ of athletes on a team being viewed as a leader (Loughead \& Hardy, 2005). Consequently, when the participants were asked to assess the behaviors of their athlete leaders, there may have been the possibility that they did not focus on the same type of leader. For instance, some athletes may have focused more on formal leaders while others may have focused more on informal leaders. Future research may want to examine these two types of athlete leaders independently. Currently, researchers do not know whether formal and informal athlete leaders exhibit similar or different leadership behaviors. Their behavior could have an impact on how coaches and practitioners develop these two types of athlete leaders on their teams.

The findings of the current study extent the athlete leadership literature from a practical perspective. The results suggest athletes' perceptions of their athlete leaders' behaviors, in particular Training and Instruction, Social Support, Democratic Behavior, and Autocratic Behavior, have important implications for influencing perceptions of task and social cohesion. Therefore, athlete leaders should be aware of how their leadership behaviors can influence aspects of the team environment 
(i.e., cohesion). Therefore, practitioners working with teams (e.g., sport psychology consultant, coaches) should consider implementing educational programs with athlete leaders on how to foster these athlete leader behaviors. From a Training and Instruction perspective, practitioners may want to emphasize to athlete leaders the importance of working hard, being able to instruct their teammates on the skills, techniques, and tactics of their respective sport. As for nurturing Social Support behaviors, practitioners should educate athlete leaders on how to develop a positive team atmosphere and the importance of having good relationships with teammates. In terms of developing Democratic Behaviors, athlete leaders need to be aware of the importance of asking the opinions and feelings of their teammates. Finally, athlete leaders need to be educated on how the use of Autocratic Behaviors (e.g., stressing high personal authority over teammates) can negatively influence perceptions of cohesion.

Although the study makes a contribution to the athlete leadership research, a few limitations should be addressed. First, the Leadership Scale for Sports (LSS; Chelladurai \& Saleh, 1980) was originally developed to assess the perceptions of coaches' leadership behaviors. Chelladurai (1998) suggested that the LSS may not assess all the dimensions of coaching behaviors. Therefore, it could be argued that not all of the athlete leader behaviors are captured by the LSS. Perhaps it would be beneficial to have a scale specific to athlete leaders and their leadership behaviors. A second limitation involves the correlational design used for the current study. Although a correlational design shows that a relationship exists between two constructs, this type of design does not allow researchers to infer cause and effect. Therefore, it is unknown as to whether the relationship is directional or cyclical in nature. A third limitation concerns the internal consistency values of the GEQ. Carron, Brawley, et al. (2002) noted that low internal consistency values should not be surprising for a dynamic multidimensional construct such as cohesion. The internal consistency values reported in the current study are similar to those reported in the initial development of the GEQ (Carron et al., 1985) and similar to those in subsequent studies (e.g., Brawley, Carron, \& Widmeyer, 1993; Hardy, Hall, \& Carron, 2003; Hausenblas \& Carron, 1996; Prapavessis \& Carron, 1996). The lower internal consistency values tend to suggest that teams may be at a specific stage in their development and thus may have unreliable cognitions concerning that particular dimension of cohesion. Future research should examine the impact that group development has on perceptions of cohesion.

The results of the current study are encouraging regarding the relationship between athlete leadership behaviors and cohesion, and there are a number of possible avenues for future research. Researchers could examine whether cohesion mediates the relationship between athlete leader behaviors and outcomes such as team performance or athlete satisfaction. Carron's (1982) conceptual model is mediational in nature and research testing this assumption has been sparse. The majority of research examining cohesion has tested direct relationships, such as the leadership-cohesion relationship. Recently, Loughead and colleagues (e.g., Loughead \& Carron, 2004; Loughead, Colman, \& Carron, 2001; Loughead, Patterson, \& Carron, 2008) have conducted several studies to determine whether cohesion acted as a mediator between fitness leader behaviors and several exercise outcomes. Taken together, the results from these studies indicated that task cohesion, in most cases Individual Attractions to the Group-Task, served to mediate the relationship between fitness leader behaviors and various exercise-related outcomes. 
While the emerging body of athlete leadership literature has provided a platform from which to further explore this type of leadership in the sports domain, it is recommended that future research continue to examine athlete leader behaviors. For instance, Chelladurai's (1993) Multidimensional Model of Leadership may be a potentially useful framework for better understanding how the various types of athlete leader behaviors influence or are influenced by various constructs. In this model, it is hypothesized that situational characteristics (e.g., task type, social norms, goals), leader characteristics (e.g., gender, maturity, experience), and member characteristics (e.g., competence in the task, need for affiliation) influence a leader's behavior. In turn, it is hypothesized that leader behavior will influence the team's performance and the athlete's satisfaction. It has been shown in previous coach leadership research that gender, personality, age, maturity, and experience are related to coaching behaviors (Chelladurai \& Carron 1981; Riemer \& Toon, 2001). In addition, situational characteristics, such as organizational goals were found to influence coaching behavior (Erle, 1981; Chelladurai, 1978). Finally, it has been shown that coaching behaviors influence both team performance and athlete satisfaction (Chelladurai, 1978; Riemer \& Toon, 2001). Although the Multidimensional Model of Leadership has been used extensively to examine coach leadership, it has yet to be fully applied to the study of athlete leadership.

The present study attempted to build upon a small body of athlete leadership research. This study used a theoretical framework and quantitative methods to examine the relationship between athlete leader behaviors and perceptions of cohesion. Overall, the results indicated that athlete leadership behaviors are related to both task and social dimensions of cohesion. It is hoped that the results from the current study may help educate researchers, sport psychology consultants, athletes, and coaches about the emergence of athlete leadership and assist them in determining which leadership behaviors should be fostered to enhance cohesion on sport teams.

\section{Acknowledgments}

The authors would like to thank the Social Sciences and Humanities Research Council of Canada (410-2008-0977) for its support of this project.

\section{References}

Arbuckle, J.L. (2008). Amos (Version 17.0). Crawfordville, FL: Amos Development Corporation. ([computer software])

Bliese, P.D. (1998). Group size, ICC values, and group-level correlations: A simulation. Organizational Research Methods, 1, 355-373.

Bliese, P.D. (2000). Within-group agreement, non-independence, and reliability: Implications for data aggregation and analysis. In K.J. Klein \& S.W.J. Kozlowski (Eds.), Multilevel theory, research, and methods in organizations: foundations, extensions and new directions (pp. 349-381). San Fransisco, CA: Jossey-Bass.

Bliese, P.D., Halverson, R.R., \& Schriesheim, C.A. (2002). Benchmarking multilevel methods of leadership: The articles, the model, and the data set. The Leadership Quarterly, $13,3-14$.

Brawley, L.R., Carron, A.V., \& Widmeyer, W.N. (1993). The influence of the group and its cohesiveness on perceptions of group goal-related variables. Journal of Sport \& Exercise Psychology, 15, 245-260. 
Carron, A.V. (1982). Cohesiveness in sport groups: Interpretations and considerations. Journal of Sport Psychology, 4, 123-138.

Carron, A.V., Brawley, L.R., \& Widmeyer, W.N. (1998). Measurement of cohesion in sport and exercise. In J.L. Duda (Ed.), Advances in sport and exercise psychology measurement (pp. 213-226). Morgantown, WV: Fitness Information Technology.

Carron, A.V., Brawley, L.R., \& Widmeyer, W.N. (2002). The Group Environment Questionnaire: Test manual. Morgantown, WV: Fitness Information Technology.

Carron, A.V., Bray, S.R., \& Eys, M.A. (2002). Team cohesion and team success in sport. Journal of Sports Sciences, 20, 119-126.

Carron, A.V., Colman, M.M., Wheeler, J., \& Stevens, D. (2002). Cohesion and performance in sport: A meta-analysis. Journal of Sport \& Exercise Psychology, 24, 168-188.

Carron, A.V., Hausenblas, H.A., \& Eys, M.A. (2005). Group dynamics in sport. Morgantown, WV: Fitness Information Technology.

Carron, A.V., Widmeyer, W.N., \& Brawley, L.R. (1985). The development of an instrument to assess cohesion in sports teams: The Group Environment Questionnaire. Journal of Sport Psychology, 7, 244-266.

Chan, D. (1998). Functional relations among constructs in the same content domain at different levels of analysis: A typology of composition models. The Journal of Applied Psychology, 83, 234-246.

Chelladurai, P. (1978). A contingency model of leadership in athletics. Unpublished doctoral dissertation, University of Waterloo, Department of Management Sciences, Ontario, Canada.

Chelladurai, P. (1993). Leadership. In R.N. Singer, M. Murphy, \& L.K. Tennant (Eds.), Handbook on research on sport psychology (pp. 647-671). New York: McMillan.

Chelladurai, P. (1998). Measurement of leadership in sport. In J.L. Duda (Ed.), Advances in sport and exercise psychology measurement (pp. 227-253). Morgantown, WV: Fitness Information Technology.

Chelladurai, P., \& Carron, A.V. (1981). Task characteristics and individual differences, and their relationship to preferred leadershipin sports. Psychology of Motor Behavior and Sport, $1982,87$.

Chelladurai, P., \& Saleh, S.D. (1980). Dimensions of leader behavior in sports: Development of a leadership scale. Journal of Sport Psychology, 2, 34-45.

Dupuis, M., Bloom, G.A., \& Loughead, T.M. (2006). Team captains' perceptions of athlete leadership. Journal of Sport Behavior, 29, 60-78.

Erle, F.J. (1981). Leadership in competitive and recreational sport. Unpublished master's thesis. University of Western Ontario, London, Ontario.

Eys, M.A., Loughead, T.M., \& Hardy, J. (2007). Athlete leadership dispersion and satisfaction in interactive sports. Psychology of Sport and Exercise, 8, 281-296.

Gardner, D.E., Shields, D.L.L., Bredemeier, B.J.L., \& Bostrom, A. (1996). The relationship between perceived coaching behaviors and team cohesion among baseball and softball players. The Sport Psychologist, 10, 367-381.

George, J.M. (1990). Personality, affect, and behavior in groups. The Journal of Applied Psychology, 75, 107-116.

Glenn, S.D., \& Horn, T.S. (1993). Psychological and personal predictors of leadership behavior in female soccer athletes. Journal of Applied Sport Psychology, 5(1), 17-34.

Hardy, J., Hall, C.R., \& Carron, A.V. (2003). Perceptions of team cohesion and athlete's use of imagery. International Journal of Sport Psychology, 34, 151-167.

Hausenblas, H.A., \& Carron, A.V. (1996). Group cohesion and self-handicapping in female and male athletes. Journal of Sport \& Exercise Psychology, 18, 132-143.

Heuzé, J.P., Raimbault, N., \& Fontayne, P. (2006). Relationships between cohesion, collective efficacy and performance in professional basketball teams: An examination of mediating effects. Journal of Sports Sciences, 24, 59-58. 
Hu, L., \& Bentler, P.M. (1998). Fit indices in covariance structure modeling: Sensitivity to underparameterized model misspecification. Psychological Methods, 3, 424-453.

Hu, L., \& Bentler, P.M. (1999). Cutoff criteria for fit indexes in covariance structure analysis: Conventional criteria versus new alternatives. Structural Equation Modeling, 6, 1-55.

Jackson, D.L., Gillaspy, J.A., \& Purc-Stephenson, R. (2009). Reporting practices in confirmatory factor analysis: An overview and some recommendations. Psychological Methods, 14, 6-23.

James, L.R. (1982). Aggregation bias in estimates of perceptual agreement. The Journal of Applied Psychology, 67, 219-229.

James, L.R., Demaree, R.G., \& Wolf, G. (1984). Estimating within-group interrater reliability with and without response bias. The Journal of Applied Psychology, 69, 85-98.

Jowett, S., \& Chaundy, V. (2004). An investigation into the impact of coach leadership and coach-athlete relationship of group cohesion. Group Dynamics, 8, 302-311.

Lott, A.J., \& Lott, B.E. (1965). Group cohesiveness as interpersonal attraction: A review of relationships with antecedent and consequent variables. Psychological Bulletin, 64, 259-309.

Loughead, T.M., \& Carron, A.V. (2004). The mediating role of cohesion in the leader behavior-satisfaction relationship. Psychology of Sport and Exercise, 5, 355-371.

Loughead, T.M., Colman, M.M., \& Carron, A.V. (2001). The effects of leadership and class cohesion on the adherence of older adult exercisers. Small Group Research, 32, 558-575.

Loughead, T.M., \& Hardy, J. (2005). An examination of coach and peer leader behaviors in sport. Psychology of Sport and Exercise, 6, 303-312.

Loughead, T.M., Hardy, J., \& Eys, M.A. (2006). The nature of athlete leadership. Journal of Sport Behavior, 29, 142-158.

Loughead, T.M., Patterson, M.M., \& Carron, A.V. (2008). The impact of fitness leader behavior and cohesion on an exerciser's affective state. International Journal of Sport and Exercise Psychology, 6, 53-68.

Nunally, J.C., \& Bernstein, I.H. (1994). Psychometric theory (3rd ed.). New York: McGrawHill.

Prapavessis, H., \& Carron, A.V. (1996). The effect of group cohesion on competitive state anxiety. Journal of Sport \& Exercise Psychology, 18, 64-74.

Raudenbush, S., Bryk, A., Cheong, Y.F., Congdon, R., \& du Toit, M. (2004). HLM 6 : Hierarchical linear and nonlinear modeling. Lincolnwood, IL: Scientific Software International.

Riemer, H.A., \& Toon, K. (2001). Leadership and satisfaction in tennis: Examination of congruence, gender and ability. Research Quarterly for Exercise and Sport, 72, 243-256.

Senécal, J., Loughead, T.M., \& Bloom, G.A. (2008). A season-long team-building intervention program: Examining the effect of team goal setting on cohesion. Journal of Sport \& Exercise Psychology, 30, 186-199.

Westre, K.R., \& Weiss, M.R. (1991). The relationship between perceived coaching behaviors and group cohesion in high school football teams. The Sport Psychologist, 5, 41-54.

Yukelson, D. (1997). Principles of effective team building interventions in sport: A direct services approach at Penn State University. Journal of Applied Sport Psychology, 9, 73-96. 